\title{
New Multifunctional Frequency Filter Working in Current-mode
}

\author{
Jaroslav Koton, Kamil Vrba \\ Brno University of Technology, Dept. of Telecommunications \\ Purkynova 118, 61200 Brno \\ JaroslavKoton@phd.feec.vutbr.cz, vrbak@feec.vutbr.cz
}

\begin{abstract}
Increasing demands on circuits with low supply voltage and low power consumption lead to the realization of analogue circuits working in the current-mode. In this paper a new topology is presented of multifunctional frequency filter SIMO (Single Input Multiple Output) working the in currentmode. The proposed circuit is realized using four second-generation current conveyors and six passive elements. A high value of quality factor can be achieved, changeable independently of the characteristic frequency. The results of sensitivity and tolerance analyses of the proposed filter are given. The properties of the designed filter were also experimentally verified.
\end{abstract}

Key words: signal processing, frequency filters, current conveyor, currentmode, autonomous circuit

\section{Introduction}

Currently research and development in the area of designing linear circuit structures is focused on the application of new active elements, such as current conveyors [1], voltage conveyors [2] or transadmittance amplifiers [3]. More often we can meet with these elements in structures working in the current-mode because of their wider frequency bandwidth. Another advantage of current-mode circuits is their higher dynamic range. Using circuits working in the voltage-mode the demand of sufficient signal to noise ratio cannot be fulfilled in low supply-voltage applications. The value of supply voltage has not such an influence on the dynamic range in current-mode circuits, as in voltage-mode circuits, and this is their main advantage.

New circuits working in the current-mode can be designed using the method of adjoint transformation to a voltage-mode prototype [4]. Although this design method is relatively fast, it does not solve the problem of decreasing supply voltage, because the active elements stay the same, mostly if operational amplifiers are used. It is better to base the design of new circuits realizing frequency filters working in the currentmode on seeking a suitable autonomous circuit, which can be extended by other active and passive elements [5], [6], [7]. 


\section{Frequency filter design}

For the design of new circuits current conveyors were used. Although these active elements are not currently industrially produced, they can be found as a part of some types of current feedback amplifiers, such as AD844, or elements labeled as OPA860 and OPA861. The current conveyor of these elements is the second-generation current conveyor $\mathrm{CCII}+$. In our department we use the universal current conveyor UCC-NIB, which was developed in cooperation with the AMI Semiconductor Centre in Brno. By a suitable connection of the branches of this integrated circuit all threeport and some more-port first class, that is with a single port $\mathrm{X}$, current conveyors can be realized.

For the design it is suitable to use the generalized current conveyor (Fig. la). The relation between branch currents and voltages is given by equations

$$
u_{\mathrm{X}}=a \cdot u_{\mathrm{Y}}, i_{\mathrm{Y}}=b \cdot i_{\mathrm{X}}, i_{\mathrm{Z}}=c \cdot i_{\mathrm{X}},
$$

where $a, b$, and $c$ are voltage or current gains between individual ports. Choosing the value of these parameters $a \in\{-1 ; 1\}, b \in\{-1 ; 0 ; 1\}, c \in\{-1 ; 1\}$ a specific type of current conveyor is determined, which has to be used for the realization itself of the proposed circuit.

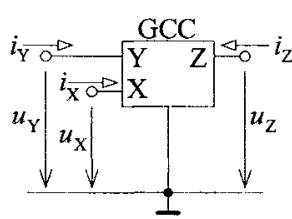

a)

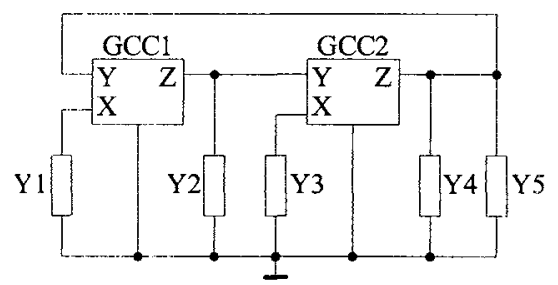

b)

Fig. 1. a) Generalized three-port current conveyor, b) autonomous circuit with two GCCs

As an initial structure for further design the autonomous circuit in Fig. 1b [7] was chosen. This circuit is described by its general characteristic equation

$$
C E=a_{1} b_{1} a_{2} b_{2} Y_{1} Y_{3}-a_{1} b_{1} Y_{1} Y_{2}-a_{2} b_{2}\left(Y_{3} Y_{4}+Y_{3} Y_{5}\right)-a_{1} c_{1} a_{2} c_{2} Y_{1} Y_{3}+Y_{2} Y_{4}+Y_{2} Y_{5}=0 \text {. }
$$

The following analysis of this circuit is focused on the realization of filters that will enable us to change the quality factor independently of the characteristic frequency. To make the realization itself as simple as possible, we will not consider any type of current conveyor as in [7], but only second-generation current conveyors, i. e. $a_{1}=a_{2}=1, b_{1}=b_{2}=0$.

Choosing the product $c_{1} c_{2}=-1$ equation (2) will simplify to the form

$$
C E=Y_{1} Y_{3}+Y_{2} Y_{4}+Y_{2} Y_{5}=0
$$

In this case all filters derived from this autonomous circuit will fulfill the condition of stability. If the character of passive elements is chosen as follows $Y_{1}=G_{1}$, 
$Y_{2}=p_{1}, Y_{3}=G_{2}$, and $Y_{4}=p_{2}+G_{3}$ (Fig. 2) then (3) will change to a form that agrees with the conditions of second-order frequency filters design

$$
\mathbf{C E}=\mathbf{p}^{2} C_{1} C_{2}+\mathbf{p} C_{1} G_{3}+G_{1} G_{2}=0
$$

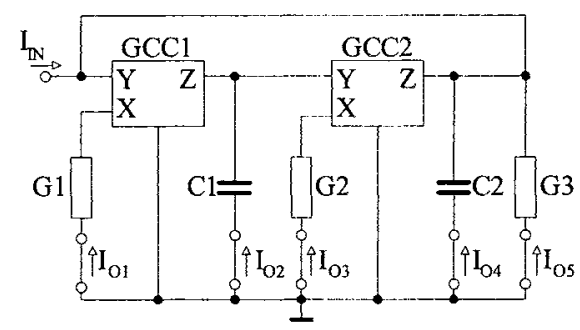

Fig. 2. Multifunctional filter working in current-mode with two $\mathrm{CC}$

Possible general current transfer functions of the circuit in Fig. 2 are

$$
\begin{gathered}
\mathbf{K}_{\mathrm{BP} 1}=\frac{\mathbf{I}_{\mathrm{O} 1}}{\mathbf{I}_{\mathrm{IN}}}=-\frac{\mathbf{p} C_{1} G_{1}}{\mathbf{C E}}, \mathbf{K}_{\mathrm{BP} 2}=\frac{\mathbf{I}_{\mathrm{O} 2}}{\mathbf{I}_{\mathrm{IN}}}=-\frac{\mathcal{c}_{1} \mathbf{p} C_{1} G_{1}}{\mathbf{C E}}, \mathbf{K}_{\mathrm{LP}}=\frac{\mathbf{I}_{\mathrm{O} 3}}{\mathbf{I}_{\mathrm{IN}}}=-\frac{c_{1} G_{1} G_{2}}{\mathbf{C E}}, \\
\mathbf{K}_{\mathrm{HP}}=\frac{\mathbf{I}_{\mathrm{O} 4}}{\mathbf{I}_{\mathrm{IN}}}=-\frac{\mathbf{p}^{2} C_{1} C_{2}}{\mathbf{C E}}, \mathbf{K}_{\mathrm{BP} 3}=\frac{\mathbf{I}_{\mathrm{O} 5}}{\mathbf{I}_{\mathrm{IN}}}=-\frac{\mathbf{p} C_{1} G_{3}}{\mathbf{C E}} \\
\mathbf{K}_{\mathrm{BR}}=\frac{\mathbf{I}_{\mathrm{O} 3}+\mathbf{I}_{\mathrm{O} 4}}{\mathbf{I}_{\mathrm{IN}}}=-\frac{\mathbf{p}^{2} C_{1} C_{2}+c_{1} G_{1} G_{2}}{\mathbf{C E}}
\end{gathered}
$$

The proposed circuit can be used for the realization of low-pass, (5c), high-pass $(5 \mathrm{~d})$, band-pass $(5 \mathrm{a}, \mathrm{b}, \mathrm{e})$, and band-reject $(5 \mathrm{f})$ frequency filters. By respecting the condition $c_{1} c_{2}=-1$ in some cases it is possible to realize either inverting or noninverting transfer function choosing the value of coefficients $c_{1}$ and $c_{2}$.

According to (4) the quality factor $Q$ and radian frequency $\omega_{0}$ are given by

$$
Q=\sqrt{\frac{C_{2}}{C_{1}}} \frac{\sqrt{G_{1} G_{2}}}{G_{3}}, \omega_{0}=\sqrt{\frac{G_{1} G_{2}}{C_{1} C_{2}}} .
$$

Relative sensitivities of these parameters to individual passive elements are

$$
\begin{gathered}
S_{\mathrm{R} C 1}^{Q}=-S_{\mathrm{R} C 2}^{Q}=-S_{\mathrm{R} G 1}^{Q}=-S_{\mathrm{R} G 2}^{Q}=-\frac{1}{2}, S_{\mathrm{R} G 3}^{Q}=-1, \\
S_{\mathrm{R} C 1}^{\omega_{0}}=S_{\mathrm{R} C 2}^{\omega_{0}}=-S_{\mathrm{R} G 1}^{\omega_{0}}=-S_{\mathrm{R} G 2}^{\omega_{0}}=-\frac{1}{2}, S_{\mathrm{R} G 3}^{\omega_{0}}=0 .
\end{gathered}
$$


If some of the passive elements $\mathrm{C}_{1}, \mathrm{C}_{2}, \mathrm{G}_{1}$ or $\mathrm{G}_{2}$ changes by $1 \%$, the quality factor $Q$ and radian frequency $\omega_{0}$ will change by $0.5 \%$ or by $-0.5 \%$. The highest sensitivity of the quality factor is to the change of the admittance $\mathrm{G}_{3}$.

Using the circuit in Fig. 2 for frequency filter design, problems can appear if a high value of quality factor is required. The analyses show that in that case it is suitable to use a low impedance of resistor $R_{3}$. This requirement can be fulfilled if the resistors $R_{1}$ and $R_{2}$ are also of low impedance. Their values cannot be arbitrarily low, because if the impedance connected to a current port $X$ is close to or even smaller than the input impedance of the port $X$, the circuit leaves its functionality at high frequencies. The value of the input impedance of the port $X$ is a very limiting factor of current conveyors and its reduction is the main problem currently solved by many researches [8-10].

The influence of the parasitic impedance of the port X can be suppressed by the modification of the circuit in Fig. 1b) by extending it with other active and passive elements. The advantage is that the resistors connected to the port $\mathrm{X}$ can a have higher value and hence the parasitic properties of the current conveyor used will not show so much.

In Fig. 3a) the modified structure of the autonomous circuit with four generalized current conveyors and six passive elements is presented.

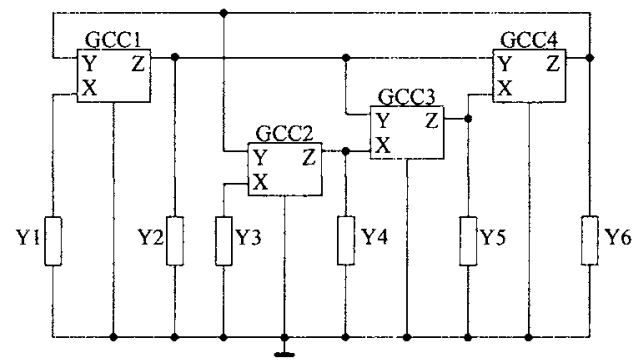

a)

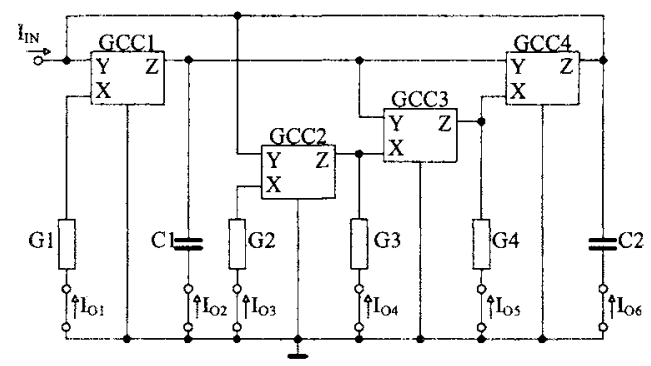

b)

Fig. 3. a) Modified autonomous circuit with four GCC, b) multifunctional filter working in current-mode

The design of the circuit starts with the general characteristic equation, as in the previous case. In the further design we will also use only second-generation current 
conveyors. The characteristic equation of the autonomous circuit in Fig. $3 \mathrm{a}$ is given by

$$
C E=c_{1} c_{3} c_{4} Y_{1} Y_{4}-c_{1} c_{4} Y_{1} Y_{5}-c_{2} c_{3} c_{4} Y_{2} Y_{3}+Y_{2} Y_{6}=0
$$

Choosing the value of the products of the coefficients $c_{1} c_{3} c_{4}=1, c_{1} c_{4}=-1$, and $c_{2} c_{3} c_{4}=-1$ and the character of passive elements $Y_{1}=G_{1}, Y_{2}=p_{1}, \quad Y_{3}=G_{2}$, $Y_{4}=G_{3}, Y_{5}=G_{4}$, and $Y_{6}=p_{2}$ it is possible to determine the type of the current transfer functions realizable by the circuit designed in Fig. $3 \mathrm{~b}$

$$
\begin{gathered}
\mathbf{K}_{\mathrm{BP} 1}=\frac{\mathbf{I}_{\mathrm{O} 1}}{\mathbf{I}_{\mathrm{IN}}}=\frac{-\mathbf{p} C_{1} G_{1}}{\mathbf{C E}}, \mathbf{K}_{\mathrm{BP} 2}=\frac{\mathbf{I}_{\mathrm{O} 2}}{\mathbf{I}_{\mathrm{IN}}}=\frac{-c_{1} \mathbf{p} C_{1} G_{1}}{\mathbf{C E}}, \mathbf{K}_{\mathrm{BP} 3}=\frac{\mathbf{I}_{\mathrm{O} 3}}{\mathbf{I}_{\mathrm{IN}}}=\frac{-\mathbf{p} C_{1} G_{2}}{\mathbf{C E}}, \\
\mathbf{K}_{\mathrm{IP} 1}=\frac{\mathbf{I}_{\mathrm{O} 4}}{\mathbf{I}_{\mathrm{IN}}}=\frac{-c_{1} G_{1} G_{3}}{\mathbf{C E}}, \mathbf{K}_{\mathrm{LP} 2}=\frac{\mathbf{I}_{\mathrm{O} S}}{\mathbf{I}_{\mathrm{IN}}}=\frac{-c_{1} G_{1} G_{4}}{\mathbf{C E}}, \mathbf{K}_{\mathrm{HP}}=\frac{\mathbf{I}_{\mathrm{O} 6}}{\mathbf{I}_{\mathrm{IN}}}=\frac{-\mathbf{p}^{2} C_{1} C_{2}}{\mathbf{C E}}, \\
\mathbf{K}_{\mathrm{LP} 3}=\frac{\mathbf{I}_{\mathrm{O} 4}+\mathbf{I}_{\mathrm{O} S}}{\mathbf{I}_{\mathrm{IN}}}=\frac{-c_{1} G_{1}\left(G_{3}+G_{4}\right)}{\mathbf{C E}}, \\
\mathbf{K}_{\mathrm{BR}}=\frac{\mathbf{I}_{\mathrm{O} 4}+\mathbf{I}_{\mathrm{O} S}+\mathbf{I}_{\mathrm{O} 6}}{\mathbf{I}_{\mathrm{IN}}}=-\frac{\mathbf{p}^{2} C_{1} C_{2}+c_{1} G_{1}\left(G_{3}+G_{4}\right)}{\mathbf{C E}},
\end{gathered}
$$

where $\mathbf{C E}=\mathbf{p}^{2} C_{1} C_{2}+\mathbf{p} C_{1} G_{2}+G_{1}\left(G_{3}+G_{4}\right)$. This circuit can be used as high-pass (10f), low-pass $(10 \mathrm{~d}, \mathrm{e}, \mathrm{g})$, band-pass $(10 \mathrm{a}, \mathrm{b}, \mathrm{c})$ or band-reject $(10 \mathrm{~h})$ frequency filter. In the case of transfers $(10 \mathrm{~b}),(10 \mathrm{~d}),(10 \mathrm{e})$, and $(10 \mathrm{~g})$ the choice of the coefficient $c_{1}$ will realize either the inverting or the non-inverting type of the transfer function of the filter. However, it is necessary to respect the conditions of the products of the coefficients $c_{1}$ to $c_{4}$.

The quality factor $Q$ and radian frequency $\omega_{0}$ are given as follows

$$
Q=\sqrt{\frac{C_{2}}{C_{1}}} \frac{\sqrt{G_{1}\left(G_{3}+G_{4}\right)}}{G_{2}}, \omega_{0}=\sqrt{\frac{G_{1}\left(G_{3}+G_{4}\right)}{C_{1} C_{2}}} .
$$

The relative sensitivities of the quality factor and radian frequency to passive elements are

$$
\begin{gathered}
S_{\mathrm{R} C 1}^{Q}=-S_{\mathrm{R} C 2}^{Q}=-S_{\mathrm{R} G 1}^{Q}=-\frac{1}{2}, S_{\mathrm{R} G 2}^{Q}=-1, \\
S_{\mathrm{R} G 3}^{Q}=\frac{1}{2} \frac{G_{3}}{G_{3}+G_{4}}, S_{\mathrm{R} G 4}^{Q}=\frac{1}{2} \frac{G_{4}}{G_{3}+G_{4}}, \\
S_{\mathrm{R} C 1}^{\omega_{0}}=S_{\mathrm{R} C 2}^{\omega_{\mathrm{g}}}=-S_{\mathrm{R} G 1}^{\omega_{0}}=-\frac{1}{2}, S_{\mathrm{R} G 2}^{\omega_{0}}=0,
\end{gathered}
$$




$$
S_{\mathrm{R} G 3}^{\omega_{0}}=\frac{1}{2} \frac{G_{3}}{G_{3}+G_{4}}, S_{\mathrm{R} G 4}^{\omega_{0}}=\frac{1}{2} \frac{G_{4}}{G_{3}+G_{4}} .
$$

The values of the relative sensitivities are analogous to those of the initial autonomous circuit. Moreover, the values of admittances $G_{3}$ and $G_{4}$ can be optimized here such that their influence on the properties of this circuit is minimized.

\section{Simulations}

Using the OrCAD - PSpice simulation program the behaviour of the proposed circuit was analyzed. The universal current conveyor UCC-N1B was used as the active element. For simulations the values of coefficients $c_{1}=c_{3}=-1, c_{2}=c_{4}=1$ were chosen, which fulfill the conditions for circuit stability.

If we choose $R_{1}=R_{3}=R_{4}=R$ and $C_{1}=C_{2}=C$, then using (11) the values of resistors are given can be calculated for the required values of $\omega_{0}$ and $Q$

$$
R=\frac{\sqrt{2}}{\omega_{0} C}, R_{2}=\frac{Q R}{\sqrt{2}} .
$$

The magnitudes of current transfer functions of the multifunctional frequency filter analyzed are given in Fig. 4. The value of the characteristic frequency considered is $f_{0}=4.5 \mathrm{MHz}$ and of the quality factor is $Q=20$. The results are given for transfer functions (10c), (10f), and $(10 \mathrm{~g})$.

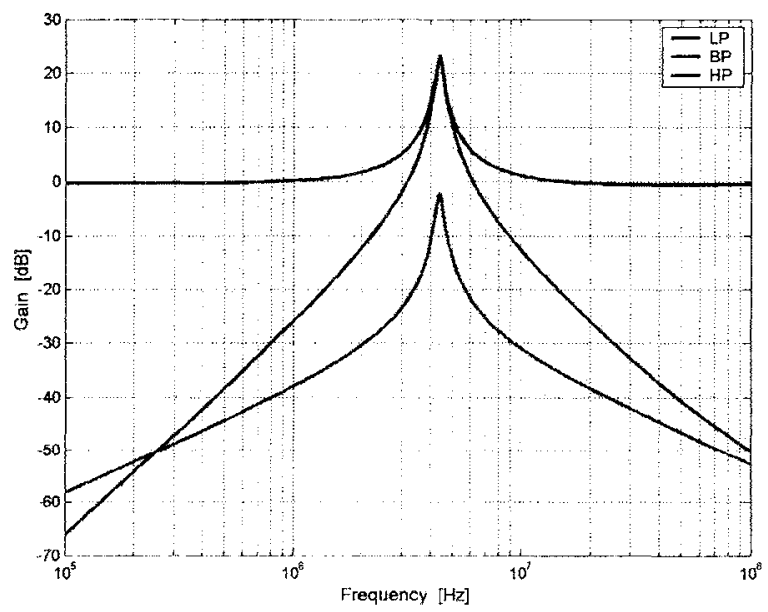

Fig. 4. Magnitudes of current transfer functions, $f_{0}=4.5 \mathrm{MHz}, Q=20$

The real properties of the active elements used cause that the quality factor is not as high as required. The current value according to simulations is just about $Q=14$. 
Also the characteristic frequency $f_{0}$ has decreased. However, it can be said that the behaviour of the designed circuit is very satisfactory. The sensitivity of the circuit to the change of passive elements is expressed by the histogram in Fig. 5. It shows the characteristic frequency shift if resistors and capacitors with $5 \%$ tolerance are used.

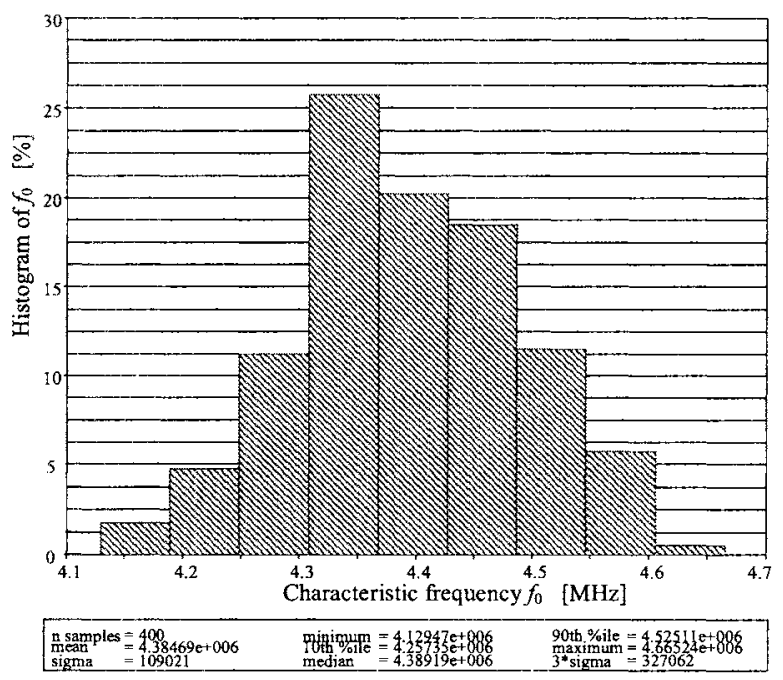

Fig. 5. Histogram of the characteristic frequency $f_{0}$ of the proposed circuit

\section{Experimental results}

The real behaviour of the designed multifunctional frequency filter was also experimentally verified. The value of characteristic frequency $f_{0}$ is $4.5 \mathrm{MHz}$. The measurements were performed for the values $147 \Omega, 210 \Omega, 2.94 \mathrm{k} \Omega$, and $14.7 \mathrm{k} \Omega$ of the resistor $R_{2}$, which according to (1la) agrees with the values $0.5,0.707,10$, and 50 of the quality factor $Q$. The results for individual current transfers are shown in Fig. 6 . The transfer functions measured were (10c), (10f), a (10g).

The value of the quality factor $Q$ is not as high as the theoretical one according to (11a), which is caused by real the properties of the circuit elements used. The best results were achieved for the low-pass frequency filter (Fig. 6c). As regards on the shape of the magnitude function, the worst of all behaviours is that of the high-pass filter, where in the frequency area of $100 \mathrm{MHz}$ a local maximum of the magnitude is formed. This can be already caused by the parasitic properties of the wiring on the PCB board. The magnitude of the transfer function realizing the band-pass frequency filter has at low frequencies a lower attenuation than expected, which is caused by the non-zero impedance of the port X [11]. This feature mostly shows in the low values of quality factor, where the impedance of resistor $R_{2}$ is close to the impedance of the port X. 


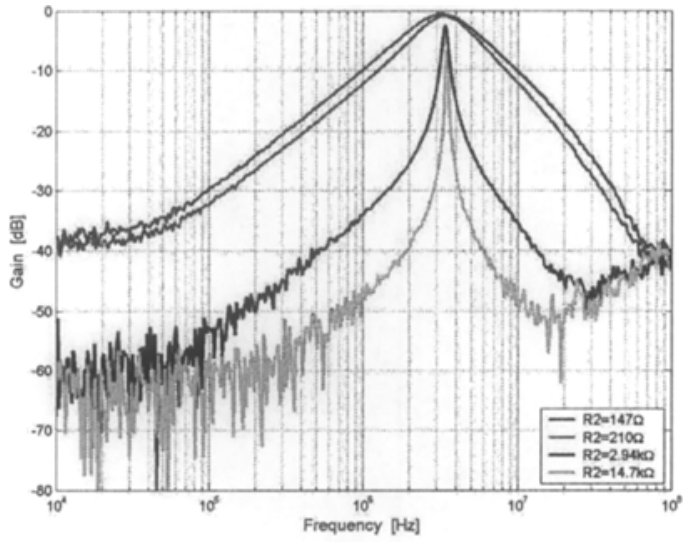

a)

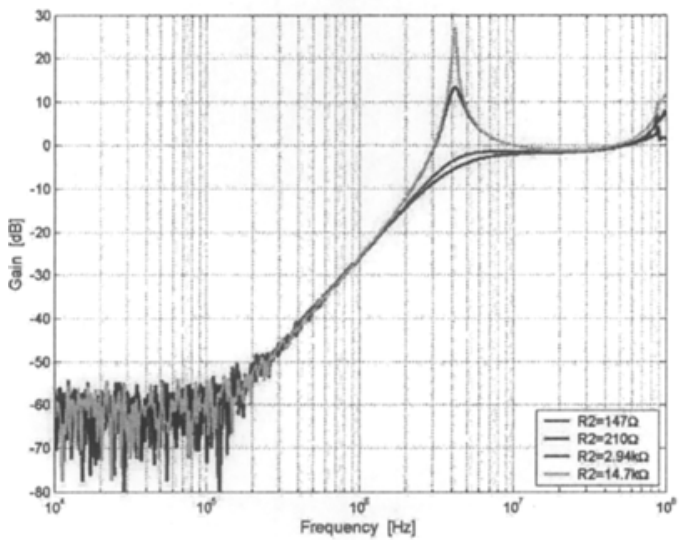

b)

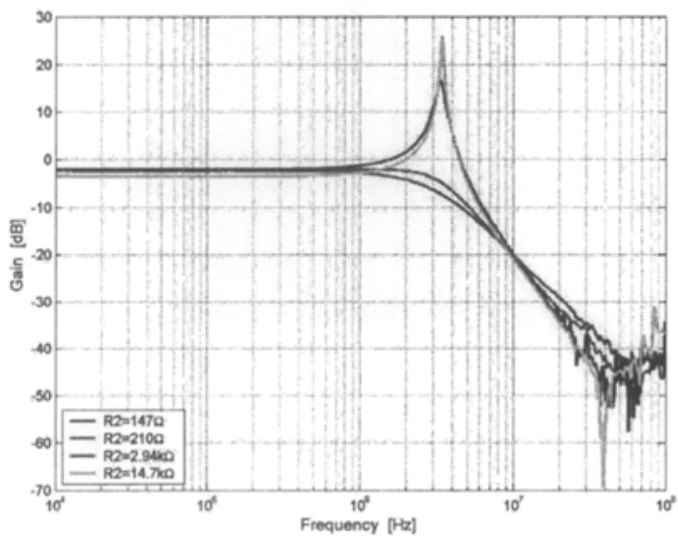

c)

Fig. 6. Measured magnitudes of transfer functions a) band-, b) high-, c) low-pass 


\section{Conclusion}

In this paper a new filtering circuit structure was proposed, which starts from an autonomous circuit. Current conveyors were used as active elements. Using this circuit it is possible to realize a second-order low-, high-, band-pass and band-reject frequency filter working in the current-mode. The circuit presented enables changing the quality factor $Q$ independently of the characteristic frequency $f_{0}$ using a single passive element. The behaviour of the designed structure was not only verified by simulations, but it was also practically realized.

\section{Acknowledgement}

The research of the current-mode using modern active elements is supported by the science project of Ministry for education MSM 0021630513 and by the Czech Science Foundation, project No. 102/06/1383.

\section{References}

[1] H. M. Hassan, A. M. Soliman: „Novel Accurate Wideband CMOS Current Conveyor“, Frequenz, 2006, Vol. 60, No. 11-12, pp. 234-236.

[2] K. N. Salama et al.: „Parasitic-Capacitance Insensitive Voltage-Mode MOSFET-C Filters Using Differential Current Voltage Conveyor"،, Circuits Systems Signal Process, 2001, Vol. 20, No. 1, pp. 1-16.

[3] Y. Sun, C. Hill, A. Szczepanski: „Large Dynamic Range High Frequency Fully Differential CMOS Transconductance Amplifier", Analog Integrated Circuits and Signal Processing, 2003, No. 34, pp. 247-255.

[4] G. W. Roberts, A. S. Sedra: "All Current-Mode Frequency Selective Circuits," Electronics Letters, 1989, Vol. 25, No. 12, pp. 759-760.

[5] J. Koton, K. Vrba, Method for Designing Frequency Filters using Universal Current Conveyors, International Transaction on Computer Science and Engineering, ISSN 17386438, 2005, Vol. 13, pp. 144-154.

[6] J. Koton, K. Vrba, P. Hanak, Frequency Filter with Current Conveyors for Signal Processing of Data-Buses Working in the Current-mode, International Conference on Networking, ICN 2006, Morne, 2006

[7] K. Vrba, J. Cajka: "Application of the General Four-port Second-kind Current Conveyor for Universal Filter Design", Electronic Journal for Engineering Technology, Vol. 5, No. 1, 2003, ISSN 1523-9926.

[8] F. Seguin, A. Fabre, New Sekond Generation Current Conveyor with Reduced Parasitic Resistance and Bandpass Filter Application, IEEE Transaction on Circuits and Systems - I, Vol. 48, No. 6, 2001, pp. 781-785.

[9] H. M. Hassan, A. M. Soliman, Novel Accurate Wideband CMOS Current Conveyor, Frequenz 60, 11-12, 2006, pp. 234-236.

[10] S. B. Salem et al., A High Performance CMOS CCII and High Frequency Applications, Analog Integr. Circ. Sig. Process, Springer Science Business Media, 2006.

[11] H. Schmidt, G. S. Moschytz, Fundamental Frequency Limitations in Current-Mode SallenKey Filters, Proceedings of the ISCAS, Monterey, California, May 31-June 3, vol. 1, pp. 57-60, 1998. 